\title{
End Effect on Determining Shear Modulus of Timber Beams in Torsion Tests
}

\author{
N. Gharavi ${ }^{\mathrm{a}}$, H. Zhang ${ }^{\mathrm{a}, *}$, Y. Xie ${ }^{\mathrm{b}}, \mathrm{T} \cdot \mathrm{He}^{\mathrm{c}}$ \\ ${ }^{a}$ School of Engineering and Built Environment, Edinburgh Napier University, 10 \\ Colinton Road, EH10 5DT, UK \\ ${ }^{b}$ Key Laboratory of Bio-based Material Science and Technology (Ministry of Education), \\ College of Material Science and Engineering, Northeast Forestry University, 26 Hexing \\ Road, Harbin, 150040, China \\ ${ }^{c}$ Department of Civil Engineering Shanghai Normal University, 100 Haisi Road, \\ Fengxian District, Shanghai, 20141, P. R. China
}

\section{Abstract}

The end effect plays an important role in determining where the rotation measuring gauges should be located in a torsion test. A thorough review on the impact of end effect in a torsion test has been conducted, followed by an experimental validation. A close-ranged photogrammetric method using binocular stereo vision technique was employed in this study. The results have indicated that the end effect has a great impact on a region of 1.5 times the cross-sectional depth of the beam from the supports. Therefore, the distance between the gauges and the supports as specified in BS EN 408:2010+A1:2012 for the torsion test setup is not sufficient for the beams with slender cross-sections. This research has also indicated that it is more appropriate to use the depth of the beam as the referencing dimension to specify this required minimum distance.

\footnotetext{
*Corresponding author

Email addresses: n.gharavi@napier.ac.uk (N. Gharavi), j.zhang@napier.ac.uk (H. Zhang), yxie@nefu.edu.cn (Y. Xie), taohe@shnu.edu.cn (T. He)
} 
Keywords: Saint-Venant's end effect, structural-size torsion test, timber beam's shear modulus, photogrammetry, binocular stereo vision

\section{Introduction}

The end effect plays an important role in the torsion test when determining the gauge distance, i.e. the distance that the gauges should be located away from the supports. Insufficient distance will bring an unnecessary error

5 into the measurement, while an excessive distance will create difficulties and inconveniences for the test setup. Hence, it is fundamentally important to investigate the end effect in this type of mechanical test. According to SaintVenant's Principle, when testing structural-sized samples the gauge sections should be located at a certain distance away from the supports or loads, where, the stress and strain in the beam are uniform and the end effect is negligible (Boresi and Schmidt, 2003; Horgan and Simmonds, 1994; Horgan and Carlsson, 2000). In the case of isotropic materials, studies by Horgan and Carlsson (2000), Horgan (1982) and Choi and Horgan (1977) have shown that Saint-Venant's end effect can be neglected at a distance approximately equal to the cross-sectional depth of the beam. However, in terms of orthotropic or transversely isotropic materials, where the longitudinal modulus of elasticity $\left(E_{L}\right)$ is far greater than its tangential $\left(E_{T}\right)$, radial modulus of elasticity $\left(E_{R}\right)$ and shear modulus $\left(G_{L T}\right)$, the latter authors proposed that the stresses $(\tau)$ decay exponentially from the location of the support or load as follows,

$$
\tau \sim C e^{-k X}
$$

where, $k \approx \frac{2 \times \pi}{h}\left(G_{L T} / E_{L}\right)^{1 / 2}$ is the decay rate; $X$ is the location from the support or load; $C$ is a constant; and, $h$ is the cross-sectional depth. Horgan 
and Carlsson (2000), Horgan (1982) and Choi and Horgan (1977) proposed that the characteristic decay length $\left(\lambda^{*}\right)$ can be determined by:

$$
\lambda^{*}=\ln (100) / k \approx \ln (100) \frac{h}{2 \times \pi}\left(\frac{E_{L}}{G_{L T}}\right)^{1 / 2}
$$

According to BS EN 338:2016: Structural Timber Strength Classes (BSI, 2016), for most of the structural timber classes for softwood, the mean longitudinal modulus of elasticity $\left(E_{L}\right)$ ranges from 0.7 to $16 \mathrm{GPa}$, while, the mean shear modulus ranges from 0.44 to $1.00 \mathrm{GPa}$. Using equation 2, it can be estimated that the decay length of the end effect is between 1.8-3 times the cross-sectional depth $(h)$ of the specimen.

The discrepancy identified indicates that the recommendation detailed in BS EN 408:2010+A1:2012: Timber structures - Structural Timber and Glued Laminated Timber - Determination of some Physical and Mechanical Properties (BSI, 2010), Clause 11.1.2 for the torsion test has limited consideration of Saint-Venant's end effect on the rotation measurements of a beam, especially for beams with a high cross-sectional aspect ratio $(h / b$, where $h$ depth and; $b$ thickness of the beam). According to this standard, the rotations should be measured at a distance of two to three times the cross-sectional thickness $(b)$ from the supports or loads. This specification is provisionally accepted for beams with low cross-sectional aspect ratios, such as a square section. However, the most commonly used timber beams in construction have slender cross-sections with aspect ratios of 3 to 5 . For these types of sections, the specified setup is clearly too close to the supporting or loading points which introduces unnecessary error in the rotation measurement.

Gupta et al. (2002b) conducted a finite element analysis of stress distribution on a timber beam subjected to a torque at one end. Their study 
indicated that a distance of two times the cross-sectional depth, plus the grip distance needs to be excluded from both ends of the beam in order to obtain a uniform shear stress distribution. Compared to the distance recommended by BS EN 408 (BSI, 2010) there is a significant difference for beams with slender cross-sections. The torsion test is widely recognised as a suitable test method for evaluating the shear properties of the timber beams as it creates a perfectly pure shear status in the specimen (Soltis and Rammer, 1994; Gupta et al., 2002a; Davalos et al., 2002; Gupta and Siller, 2005; Khokhar et al., 2008), therefore, it is important to investigate how the end effect propagates from the ends of the beam. To provide proper guidelines for the industry in measuring the shear properties using the torsion test method, there is a need to better understand the Saint-Venant's end effect in torsion test. The aim of this study is to experimentally evaluate the propagation of this effect along the beam using a tailor-made and close-range photogrammetric technique. To achieve this aim, two objectives were targeted as follows:

1. To evaluate the end effect in a torsion test method when determining the shear modulus of a timber beam and to improve the current practice adopted by the industry;

2. To develop a state-of-art photogrammetric technique for determining the shear modulus using torsion test method and to circumvent the limitation of the test setup recommended by the current code of practice.

70

Compared to the proposed photogrammetric technique, the conventional inclinometers or modified inclinometers as indicated in BS EN 408 (BSI, 2010) possess several limitations. For instance, they are limited to record only the 
rotation of a specific plane at a predetermined location. Hence, it is difficult to use these types of devices/designs to measure the distribution of the rotation on a specimen during the test. Therefore, a more advanced and accurate close-range photogrammetry technology based on stereo vision was employed to capture this rotation. Using the triangulation algorithm, the displacement of any point in the cameras' field-of-view can be measured with two or more sets of photos taken before and after the loading. A well-known method for acquiring the 3-D coordinates of an object is Binocular Stereo Vision. This is a passive triangulation technique where two images taken from two different viewpoints are analysed to extract a depth map of the scene (Peng and Guo-Qiang, 2010; Wohler, 2013). This system is similar to simplified human visual perception. Unlike the early version of this system where some setups are very strict, such as the cameras having to be mounted exactly parallel to each other, the most up-to-date system offers more flexibility and is capable of handling the camera/lens imperfections more easily.

\section{Materials and Methods}

\subsection{Materials}

With a view to that the cross-sectional aspect ratio is an important parameter in determining the impact of the end effect, 12 timber beams with the aspect ratios ranging from 1 to 4.89 were selected and tested. All beams are rectangular, structural-sized and kiln dried with a testing length of at least 19 times the largest cross-sectional dimension. Based on BS EN 408 (BSI, 2010), the samples were conditioned at the standard environment of $20^{\circ} \mathrm{C} \pm 2^{\circ} \mathrm{C}$ and $65 \% \pm 5 \%$ relative humidity for about four weeks before test- 
Table 1: Material information ${ }^{\mathrm{a}}$

\begin{tabular}{ccccccc}
\multicolumn{3}{c}{ Nominal Dimensions } & & & & \\
\cline { 1 - 3 } $\mathrm{b}$ & $\mathrm{h}$ & $\mathrm{L}$ & QTY & A.R. & M.C. & Specie \\
{$[\mathrm{mm}]$} & {$[\mathrm{mm}]$} & {$[\mathrm{m}]$} & & & {$[\%]$} & \\
\hline & & & & & & \\
95 & 95 & 1.9 & 2 & 1 & 12.8 & $\mathrm{RP}$ \\
45 & 95 & 1.9 & 2 & 2.11 & 10.4 & $\mathrm{RP}$ \\
75 & 225 & 4.3 & 2 & 3 & 11.2 & $\mathrm{WW}$ \\
45 & 170 & 3.4 & 2 & 3.78 & 10.4 & $\mathrm{WW}$ \\
45 & 195 & 3.9 & 2 & 4.33 & 12 & $\mathrm{RP}$ \\
45 & 220 & 4.3 & 2 & 4.89 & 12.5 & $\mathrm{WW}$ \\
\hline
\end{tabular}

${ }^{\text {a }}$ A.R. $=$ Aspect Ratio $(h / b)$; M.C. $=$ Moisture Content; $\mathrm{RP}=$ Redwood (Pine); WW= WhiteWood (Spruce);

ing. The moisture content of the samples was measured in accordance with BS EN 13183-1:2002, Moisture content of a piece of sawn timber. Determination by oven dry method (BSI, 2002), the results and beams specifications 100 are presented in Table 1.

\subsection{Methods}

In the example of the torsion test setup, illustrated in BS EN 408 (BSI, 2010), the gauges and rotation measuring system are not well designed. The 
circular gauge may not rotate the same angle as the specimen due to the

possible warping in the cross-section (Figure 1). In addition, the LVDTs used in the system will not be able to handle a slightly larger rotations. Therefore, there is a need to develop a more suitable method to overcome the above limitations.

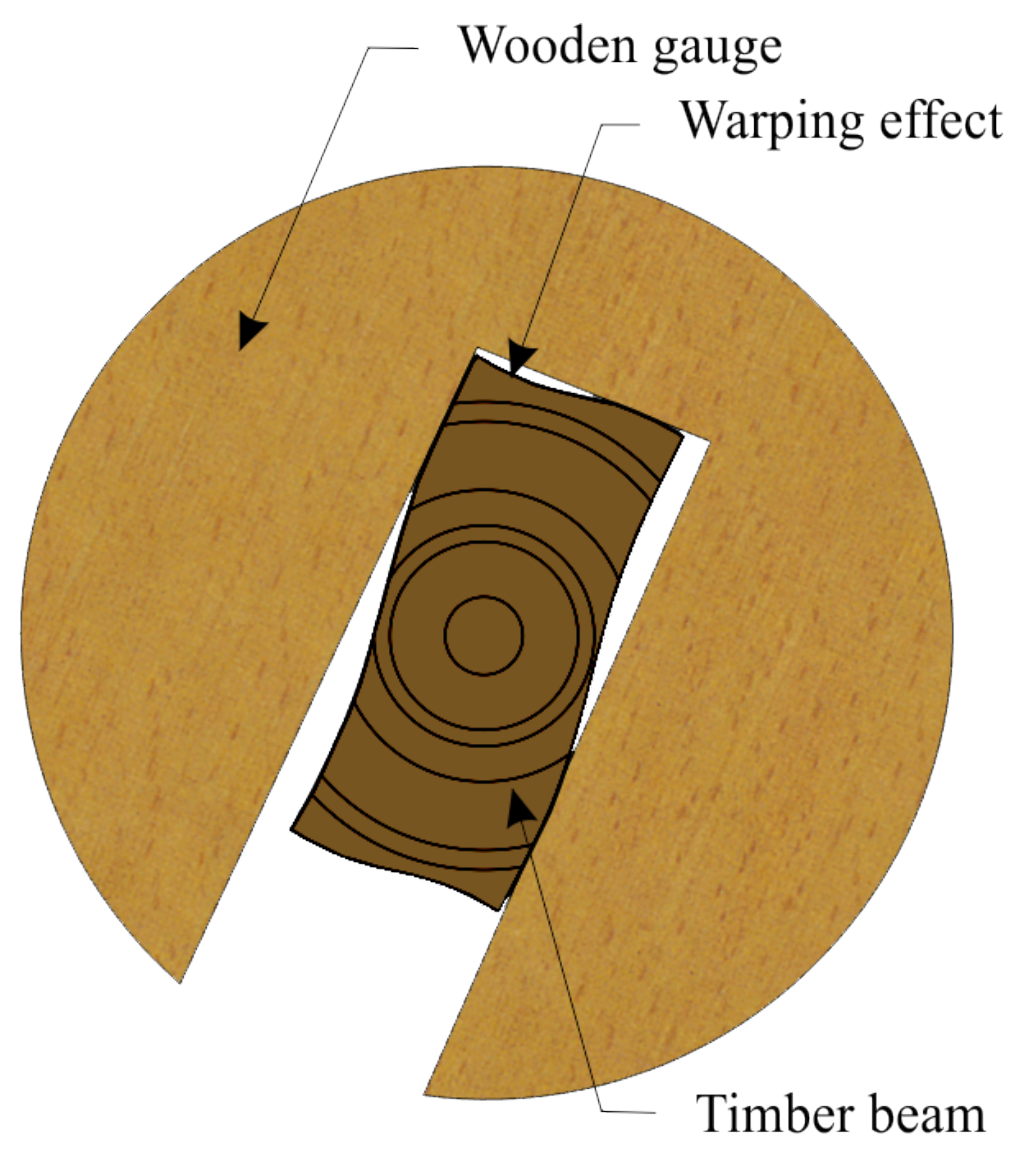

Figure 1: Circular wooden gauge and possible warping effect 


\subsubsection{Stereo Vision System}

Humans and most animal's visual perception are through a highly sophisticated 3D vision system. The binocular stereo vision system is able to compute disparity, distance and 3D coordinates of any object by simulating the human eyes. In this system, two cameras simultaneously capture the images of an object from different positions and angles (Li et al., 2016). The basic principle behind the employed binocular stereo vision is illustrated in Figure 2.

In the Figure 2, assume oxyz is the cameras coordinate systems (CCS), XOY is left and right image coordinate system and $f$ is the effective focal length, which is the distance between camera coordinate system (lenses) and image coordinate system (image sensor within the camera). Parameters that are related to left and right images are subscripted by $l$ and $r$, respectively. For convenience, the world coordinate system (WCS) is made of the left camera coordinate system $\left(\right.$ oxyz $\left.=o_{l} x_{l} y_{l} z_{l}\right)$.

The spatial positional relationship between the world coordinate system and right camera coordinate system can be obtained as:

$$
\left[\begin{array}{l}
x_{r} \\
y_{r} \\
z_{r}
\end{array}\right]=M\left[\begin{array}{l}
x \\
y \\
z \\
1
\end{array}\right]=\left[\begin{array}{llll}
r_{11} & r_{12} & r_{13} & t_{x} \\
r_{21} & r_{22} & r_{23} & t_{y} \\
r_{31} & r_{32} & r_{33} & t_{z}
\end{array}\right]\left[\begin{array}{l}
x \\
y \\
z \\
1
\end{array}\right]
$$

Where $M=[R \mid T],[R]$ expresses the rotation matrix and $[T]$ is translation transform vector between world coordinate system (oxyz) and right camera 


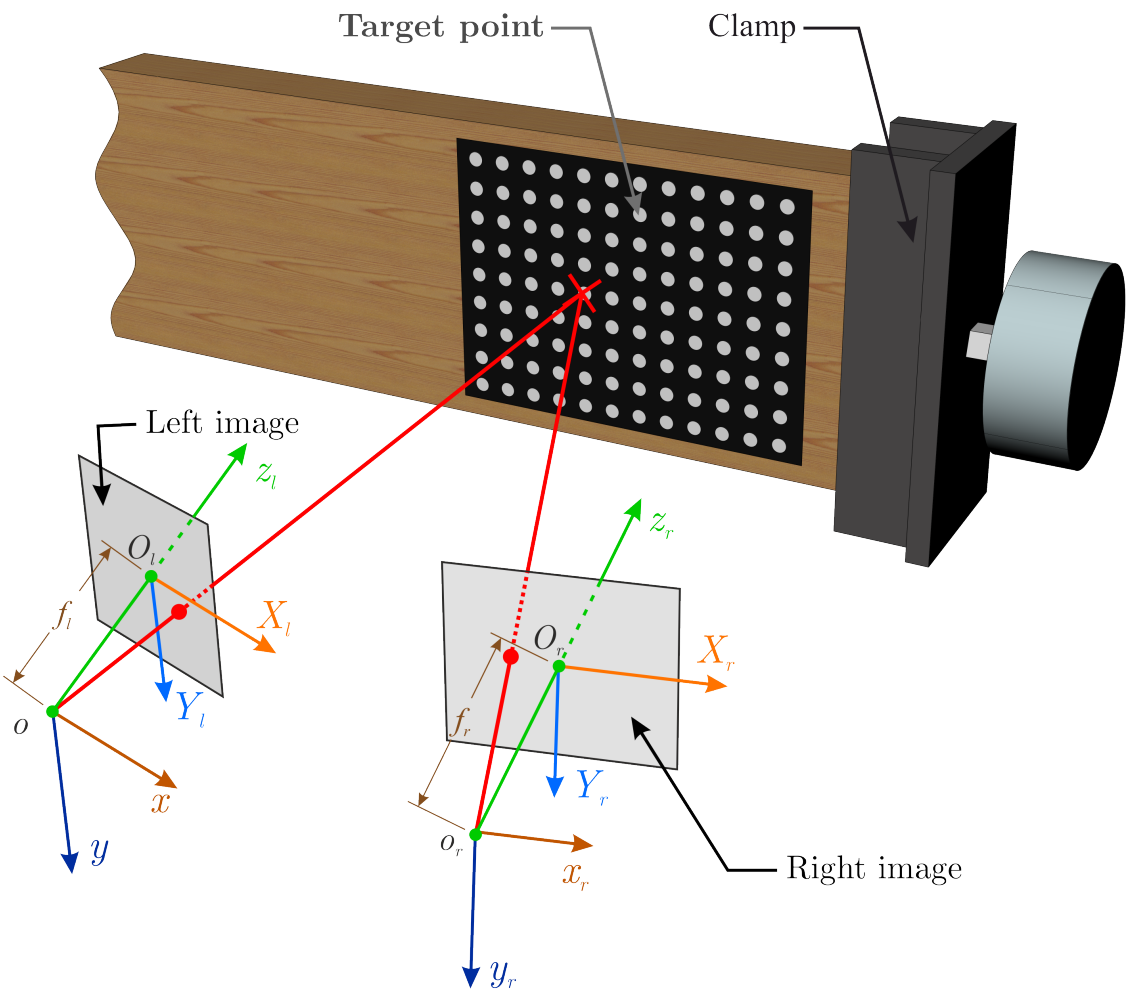

Figure 2: Principle of employed stereo vision 
coordinate system $\left(o_{r} x_{r} y_{r} z_{r}\right)$;

$$
R=\left[\begin{array}{lll}
r_{11} & r_{12} & r_{13} \\
r_{21} & r_{22} & r_{23} \\
r_{31} & r_{32} & r_{33}
\end{array}\right], T=\left[\begin{array}{c}
t_{x} \\
t_{y} \\
t_{z}
\end{array}\right]
$$

According to the pinhole imaging theory (Wohler, 2013), the following expressions can be made,

$$
s_{l}\left[\begin{array}{c}
X_{l} \\
Y_{l} \\
1
\end{array}\right]=\left[\begin{array}{ccc}
f_{l} & 0 & 0 \\
0 & f_{l} & 0 \\
0 & 0 & 1
\end{array}\right]\left[\begin{array}{c}
x \\
y \\
z
\end{array}\right], \quad s_{r}\left[\begin{array}{c}
X_{r} \\
Y_{r} \\
1
\end{array}\right]=\left[\begin{array}{ccc}
f_{r} & 0 & 0 \\
0 & f_{r} & 0 \\
0 & 0 & 1
\end{array}\right]\left[\begin{array}{c}
x_{r} \\
y_{r} \\
z_{r}
\end{array}\right]
$$

For the spatial point in the WCS, the corresponding coordinates in the left and right image can be obtained as;

$$
\rho\left[\begin{array}{c}
X_{r} \\
Y_{r} \\
1
\end{array}\right]=\left[\begin{array}{cccc}
f_{r} r_{11} & f_{r} r_{12} & f_{r} r_{13} & f_{r} t_{x} \\
f_{r} r_{21} & f_{r} r_{22} & f_{r} r_{23} & f_{r} t_{y} \\
r_{31} & r_{32} & r_{33} & t_{z}
\end{array}\right]\left[\begin{array}{c}
x \\
y \\
z \\
1
\end{array}\right]
$$

and finally, the 3D coordinate of the object can be obtained as;

$$
\left\{\begin{aligned}
x & =z \frac{X_{l}}{f_{l}} \\
y & =z \frac{Y_{l}}{f_{l}} \\
z & =\frac{f_{l}\left(f_{r} t_{x}-X_{r} t_{z}\right)}{X_{r}\left(r_{31} X_{l}+r_{32} Y_{l}+r_{33} f_{l}\right)-f_{l}\left(r_{11} X_{l}+r_{12} Y_{l}+r_{13} f_{l}\right)} \\
& =\frac{f_{l}\left(f_{r} t_{y}-Y_{r} t_{z}\right)}{Y_{r}\left(r_{31} X_{l}+r_{32} Y_{l}+r_{33} f_{l}\right)-f_{l}\left(r_{21} X_{l}+r_{22} Y_{l}+r_{23} f_{l}\right)}
\end{aligned}\right.
$$

In the equation (7), if each camera's focal length $\left(f_{l}\right.$ and $\left.f_{r}\right)$ and coordinates of the spatial point in both left and right images are known, the 
3D coordinates of the point in WCS can be obtained. Rotation matrix and translation transform vector can be determined by calibration.

The employed stereo vision setup is illustrated in Figure 3. When the stereo vision system is employed to determine 3D coordinates, camera calibration is usually required to determine the relationship between the camera lens and the image planes and also the relationship between the cameras and the calibration plate (Wohler, 2013). The overall performance of this system strongly depends on the accuracy of the calibration process. In this experiment, $100 \mathrm{~mm} \times 100 \mathrm{~mm}$ high precision ceramic calibration plate was used in the calibration process to provide a satisfactory accuracy.

The first step of the image processing in this experiment is to select the regions of interest based on the grey scale of the target area (Figure 5). Within the selected region, the target dots are located using a pattern recognition filter (roundness and size). The matrix of the centres of the target dots provides the contour of the surface deformation of the timber beam, from which rotation at the various location can be extracted and analysed (Figure $6)$.

\subsubsection{Torsion Test}

The torsion test procedure followed BS EN 408 (BSI, 2010). Beams were clamped at the supports spanning at least $19(\approx 20)$ times the largest crosssectional dimension $(h)$. Each specimen were mounted securely edgewise with vice-like grip at the supports and the centres of specimen ends were in line with the centre of rotation to allow it to rotate about its longitudinal axis (Figure 7). A gap of approximately $5 \mathrm{~mm}$ between the grip and end of the specimen was provided to allow for possible warping and prevent any 


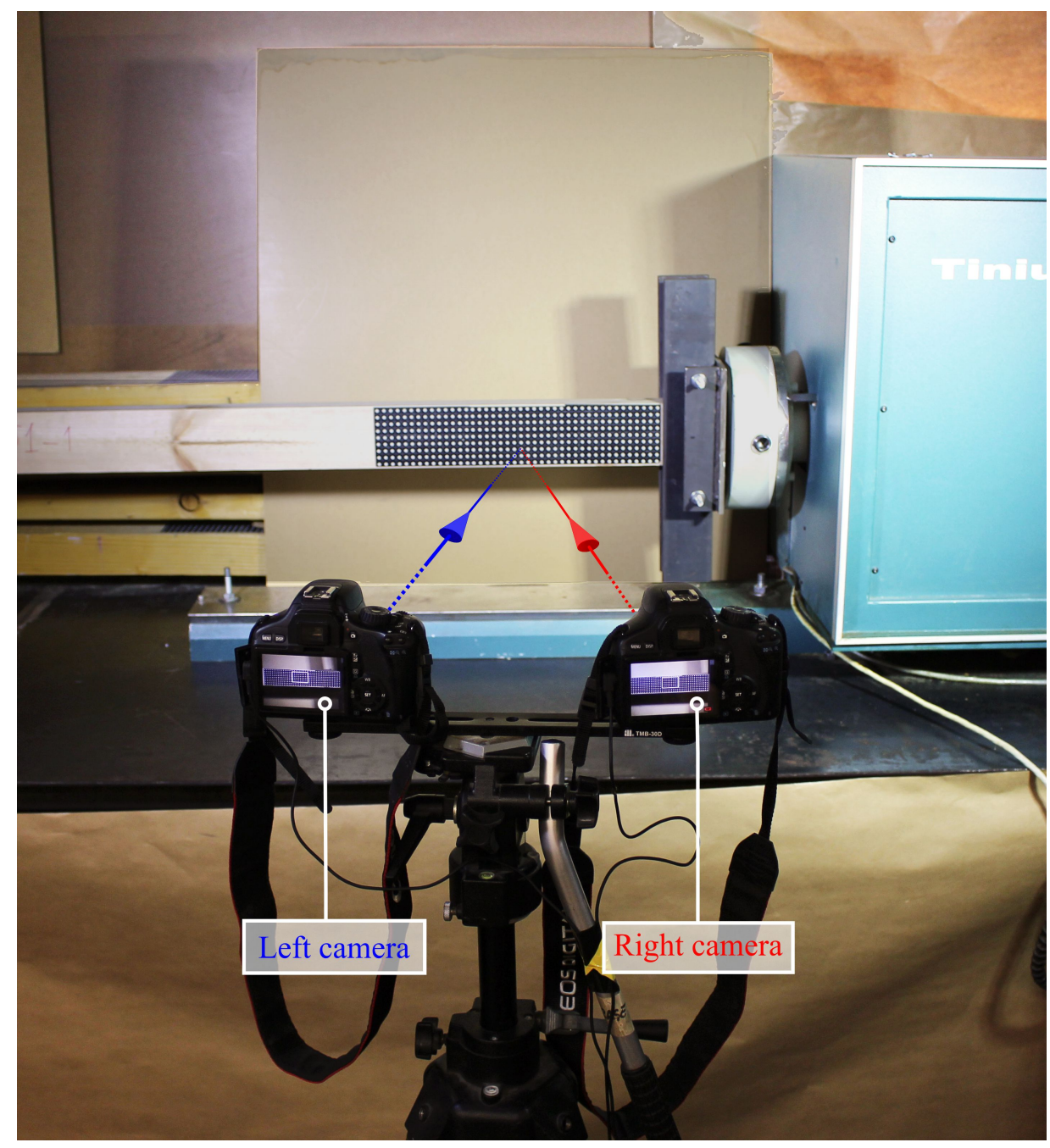

Figure 3: Binocular stereo vision setup 


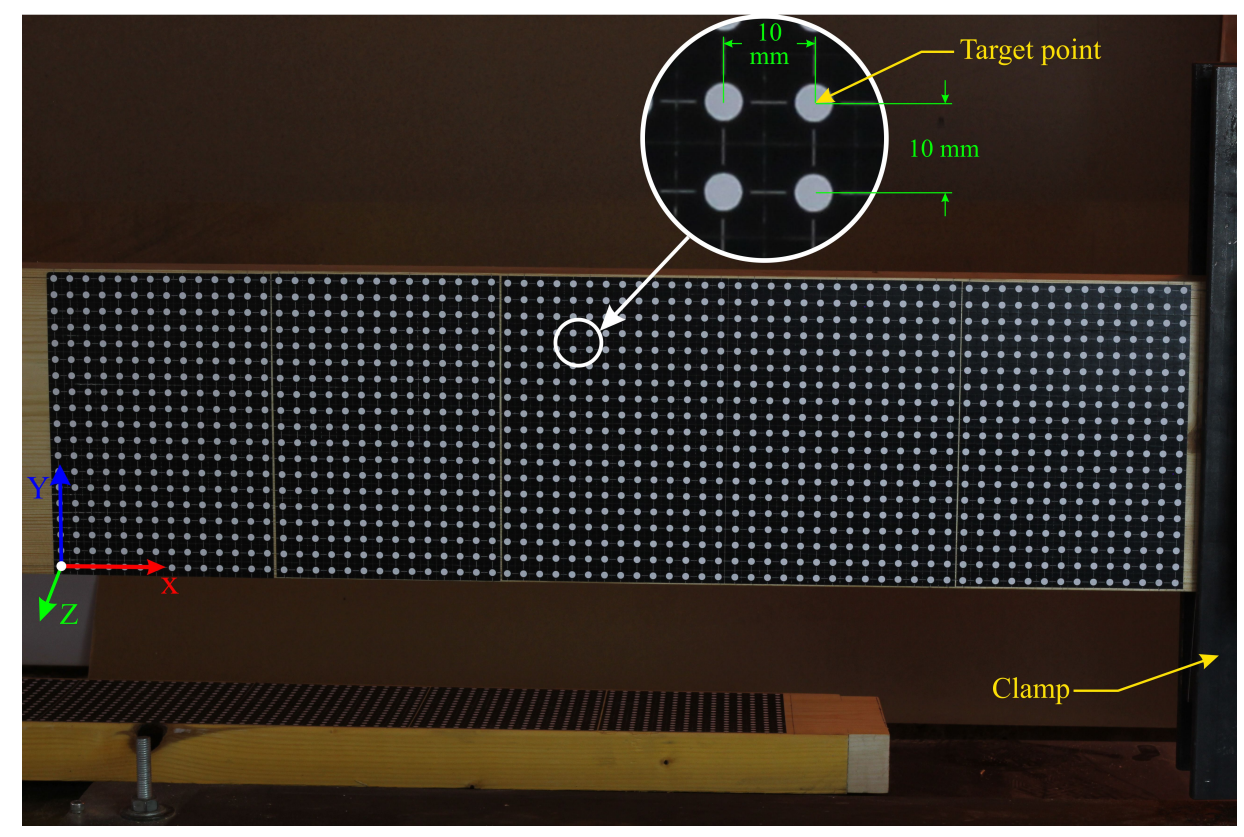

Figure 4: The image before processing

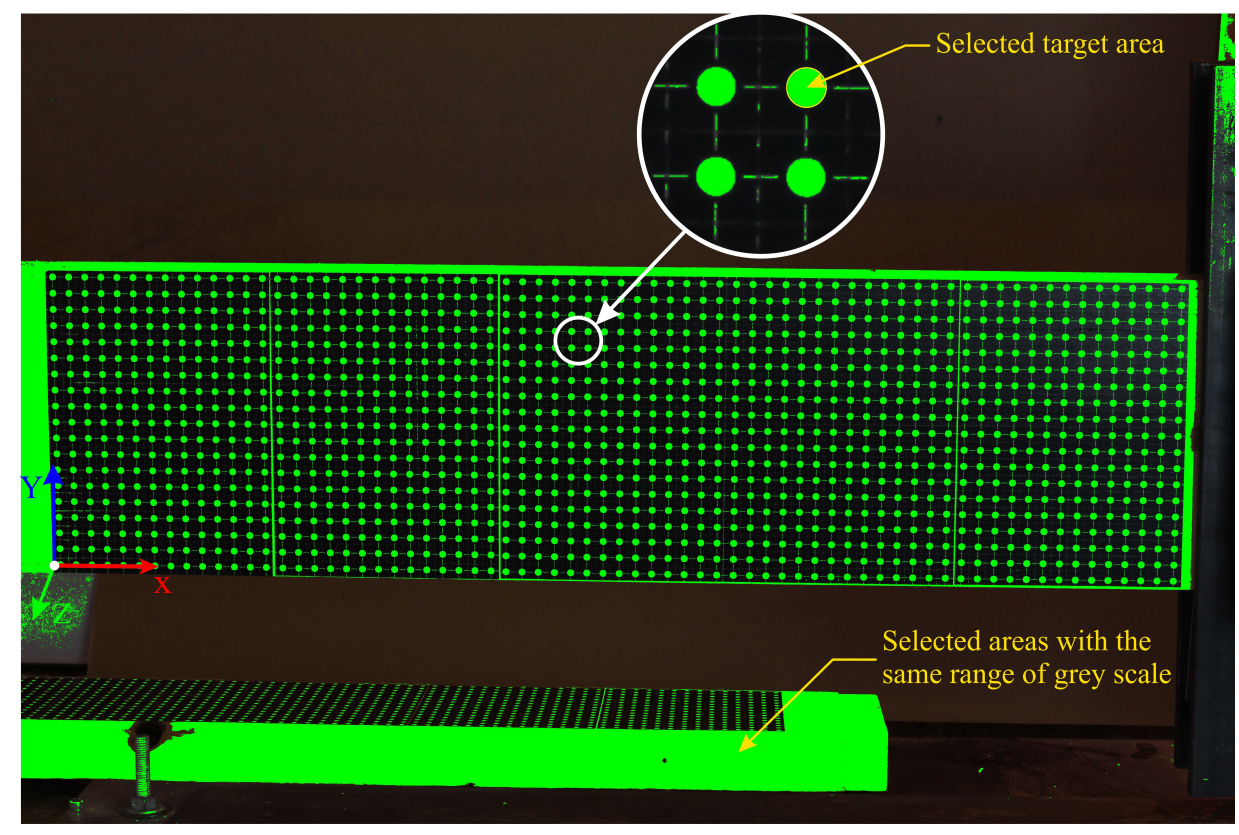

Figure 5: Image segmentation using global threshold 


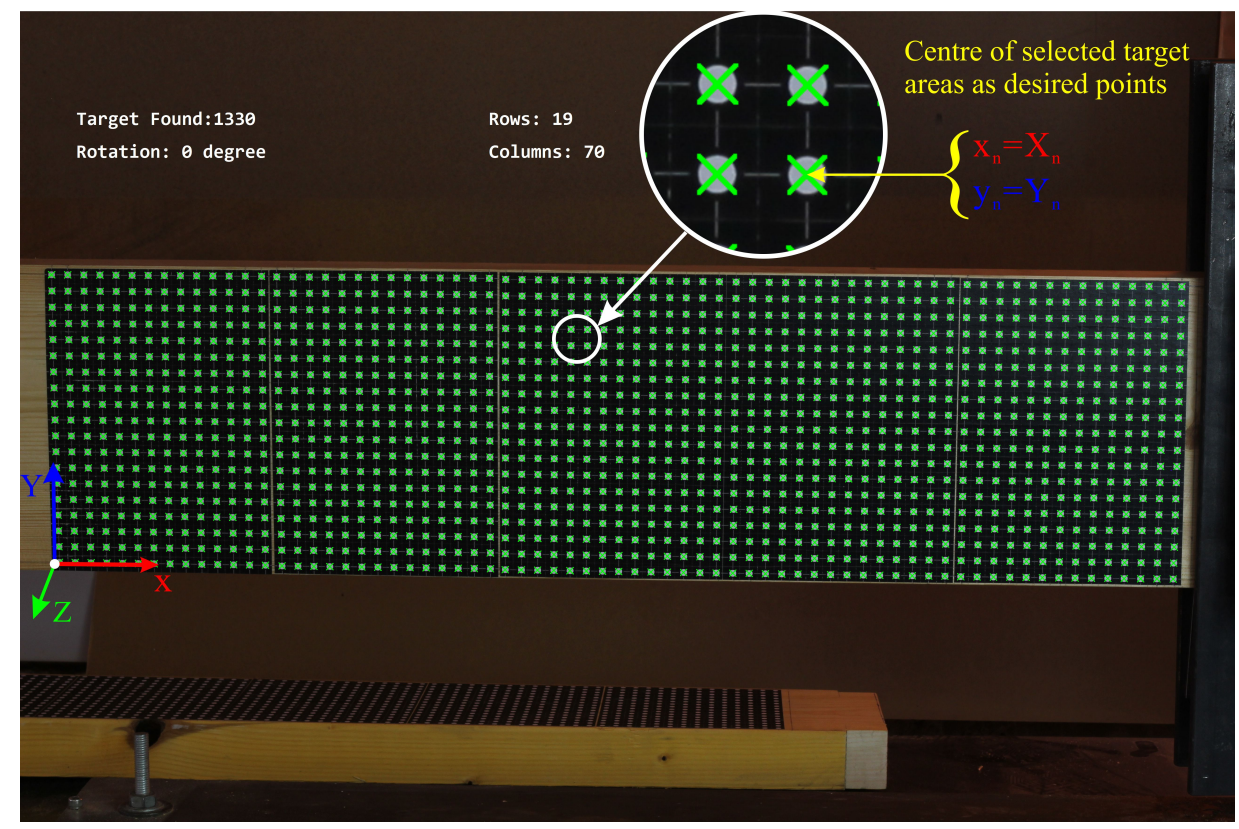

Figure 6: The target dots' matrix

build up contact stresses.

Generally speaking, over-exposed images present fewer details and as the consequence, this will reduce the accuracy of the measurement. Therefore, a dark background was used in these tests. The matrix of white target dots with $10 \mathrm{~mm}$ horizontal and vertical spacing was mapped onto this background (Figure 4). This design was printed on the glossy adhesive-backed paper and fixed to the desired areas on the surface of each beam. The glued paper was then cut with a sharp knife along each grid lines that lie between dots so that each dot can move individually. The cameras selected for this project were four Canon EOS 550Ds, which are an 18.0 megapixel digital single-lens reflex camera, and equipped with 50mm Canon EF f/1.8 II fixed focal lenses. The testing procedure was conducted using a $1 \mathrm{kN}-\mathrm{m}$ Tinius Olsen torsional 


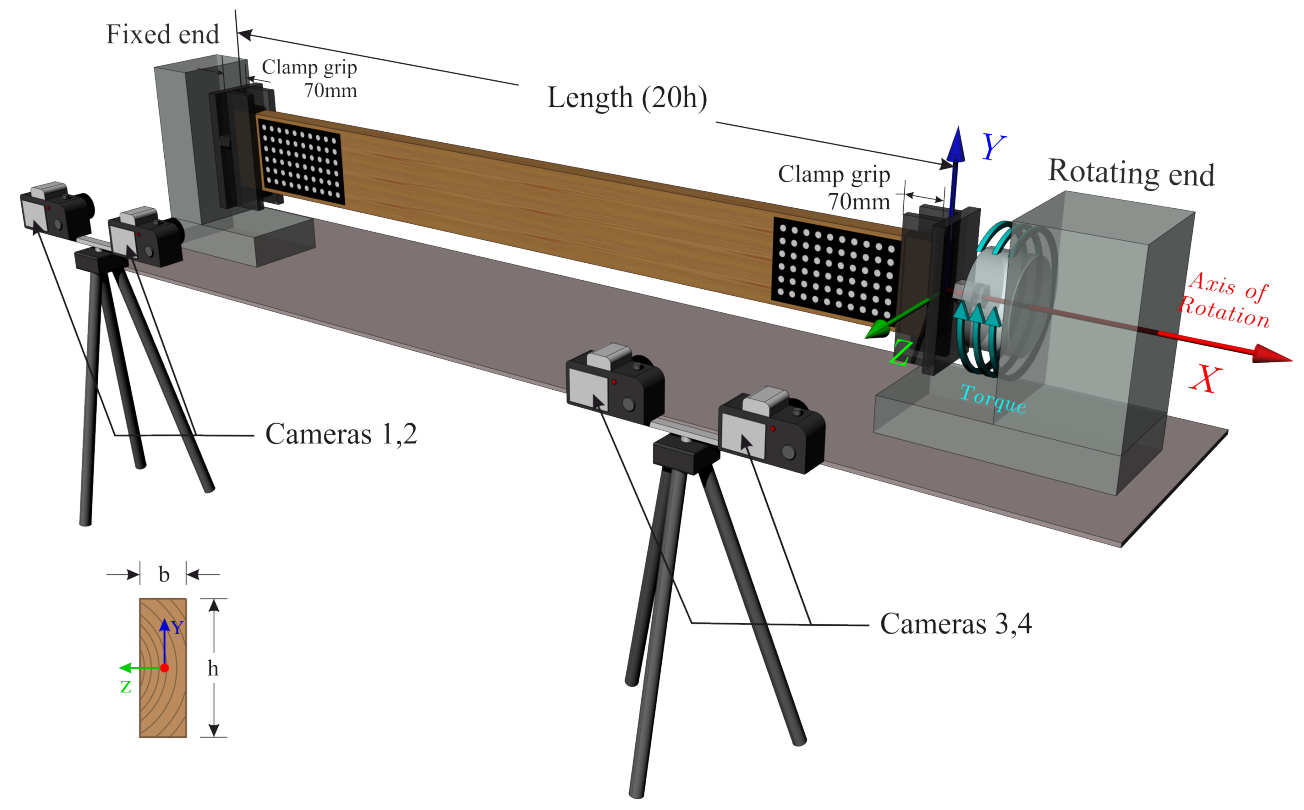

Figure 7: Torsion Test Setup

testing machine. Torque was applied to each specimen at one end with a rate of $4^{\circ}$ per min. The test setup is shown in Figure 9. Coordinates of each target point are computed in the image processing software (MvTec HALCON). This set of data profiles the surface deformation of the timber beam. By tracing the coordinates of each vertical lines, the rotation of each section can be computed using linear regression algorithm with the MATLAB programme developed by the authors. Each specimen was pre-loaded to $4^{\circ}$ rotation as an initial (reference) position and all the subsequent coordinates and forces were referenced to this position. The torque was increased at a speed of $4^{\circ}$ per min. Loading curve is shown in Figure 8. The cameras have been taking the images at constant intervals to allow us to record the torque and the rotation relationship at every vertical target lines (sections). 


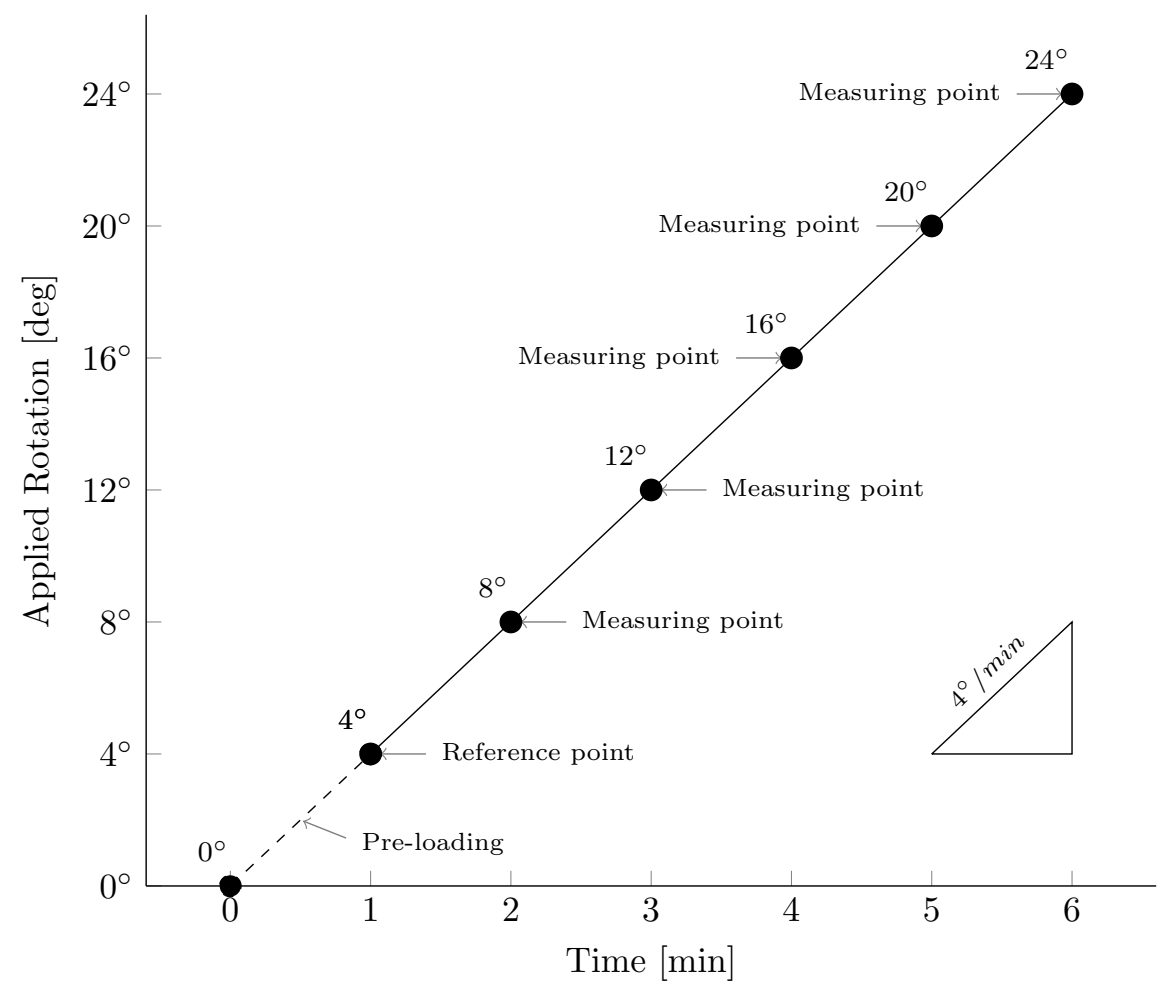

Figure 8: Applied rotation-time curve 


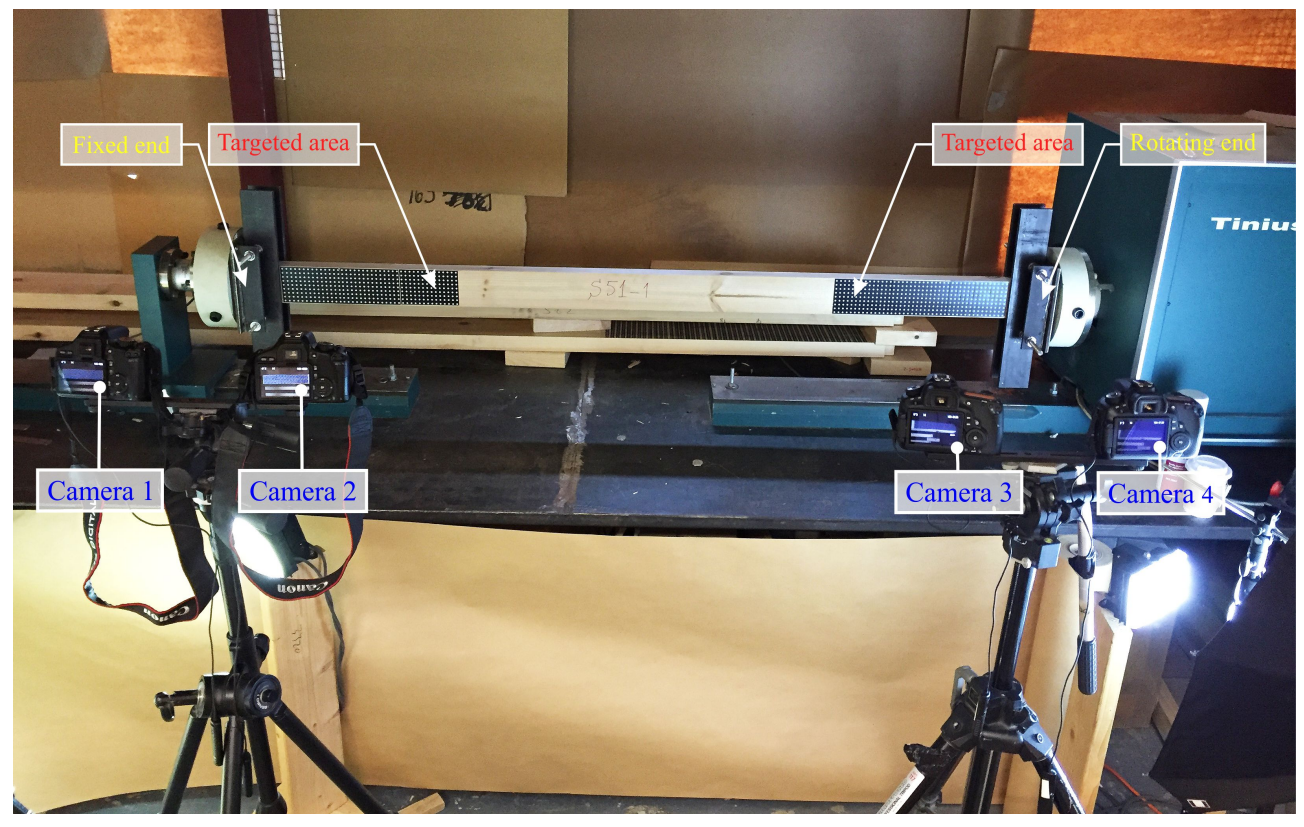

Figure 9: Torsion test setup with stereo vision system

From this relationship, the torque stiffness of each vertical section can be calculated. The corresponding shear modulus of each vertical section can then be determined using the Equation 8 based on Saint-Venant's torsion theory;

$$
G_{t o r}=\frac{k_{t o r}}{\eta h b^{3}} l_{1}
$$

Where $k_{t o r}=T / \phi_{i}$ is the torque stiffness, $T$ is applied torque, $\phi_{i}$ is relative rotation between each symmetrical pair of vertical sections $(i), G_{t o r}$ is the shear modulus and $l_{1}, \eta, h$ and $b$ are: free testing length, shape factor, crosssectional height and thickness of the beam, respectively.

The calculated shear modulus at each vertical section is plotted against the distance of this section from the end. Results are presented in Figures 10 to 15. 


\section{Results and Discussion}

The target areas are arranged symmetrically at both ends (Figure 9), and the relative rotation between each symmetrical pair of vertical lines/sections is obtained through the proposed approach. The corresponding shear modulus can be derived from the obtained relative rotations. Based on SaintVenant's principle, the end effect will have an impact on the shear modulus measurements at the gauge sections which are not sufficiently far enough away from the ends. The variation of the shear modulus measurements at these gauge sections will reveal the impact of the end effect. These variations against the distance from the end of the beam are presented in Figures 10 to 15 for all tested specimen. The Figures illustrate the decay of the end effect from the ends towards the centre. For convenience, the abscissas on the top and bottom are plotted in terms of depth and thickness coefficients. Each Figure is divided into two segments; segment one has a high variation in the shear modulus measurement due to the significant impact of the end effect, whereas segment two possesses low variation, implying considerably low impact.

It is interesting to note that the specimen with a very low aspect ratio (i.e. $\mathrm{AR}=1$ ) has relatively higher variation in both segment one and two compare to those in other specimens (Figures 10 to 15). The author's opinion is that this discrepancy is due to the fact that the torsional effect of the transverse bending loads travels longer distances in specimens with low aspect ratios. According to the torsional test setup detailed in BS EN 408 (BSI, 2010), the distance of the gauges from the ends of the beam is two to three times cross-sectional thickness $\left(2 b \leq l_{1} \leq 3 b\right)$. The results revealed that this dis- 


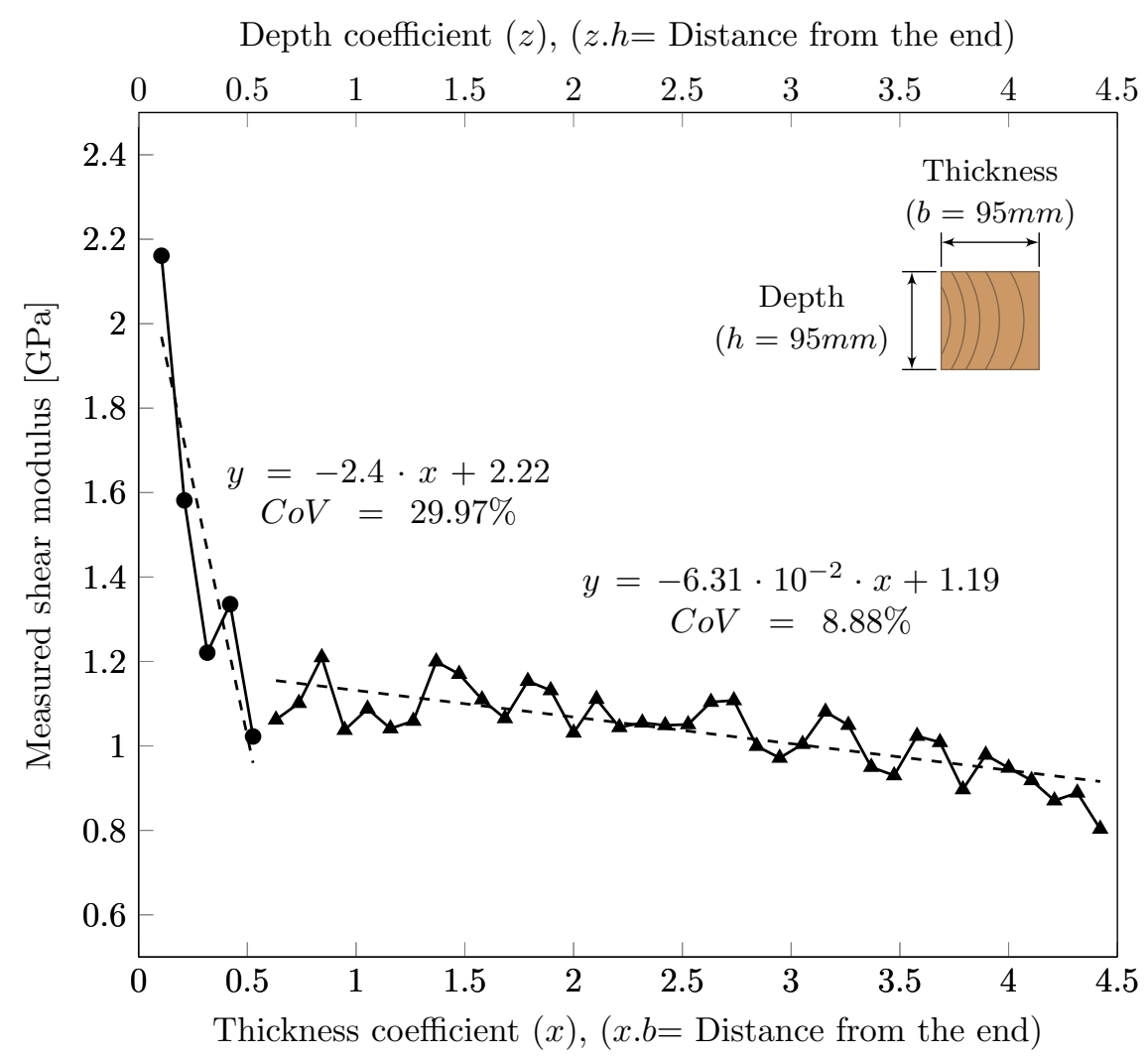

Figure 10: $\mathrm{AR}=1 ; \mathrm{CoV}=32.54 \%$; Red Pine

tance is clearly not far enough to avoid the impact of the end effect in the shear modulus measurement. This experimental study agrees with the conclusions drawn from previous research; Gupta et al. (2002b) recommended $l_{1} \geq(2 h+$ grip distance $)$ and Horgan and Simmonds (1994) recommended $l_{1} \geq(1.8 h-3 h)$, through numerical and analytical study respectively.

Arguably, there are more research support the depth of the specimen as a reference dimension in specifying the decay length of the end effect, rather than its thickness. It is beneficial for us to compile a comparison using our test results to verify this assumption. Since the impact of end effect is 


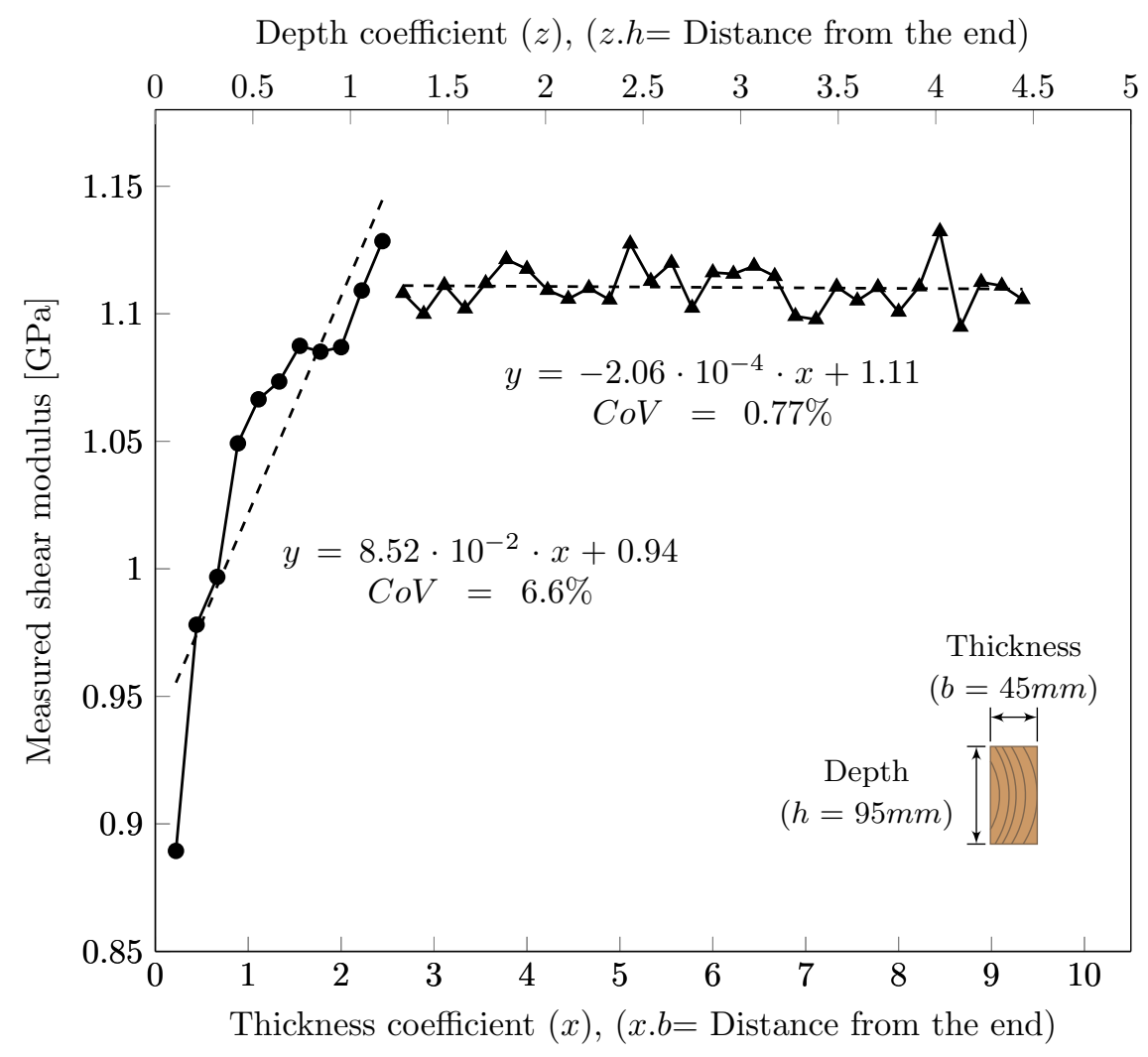

Figure 11: $\mathrm{AR}=2.11 ; \mathrm{CoV}=6.8 \%$; Red Pine 


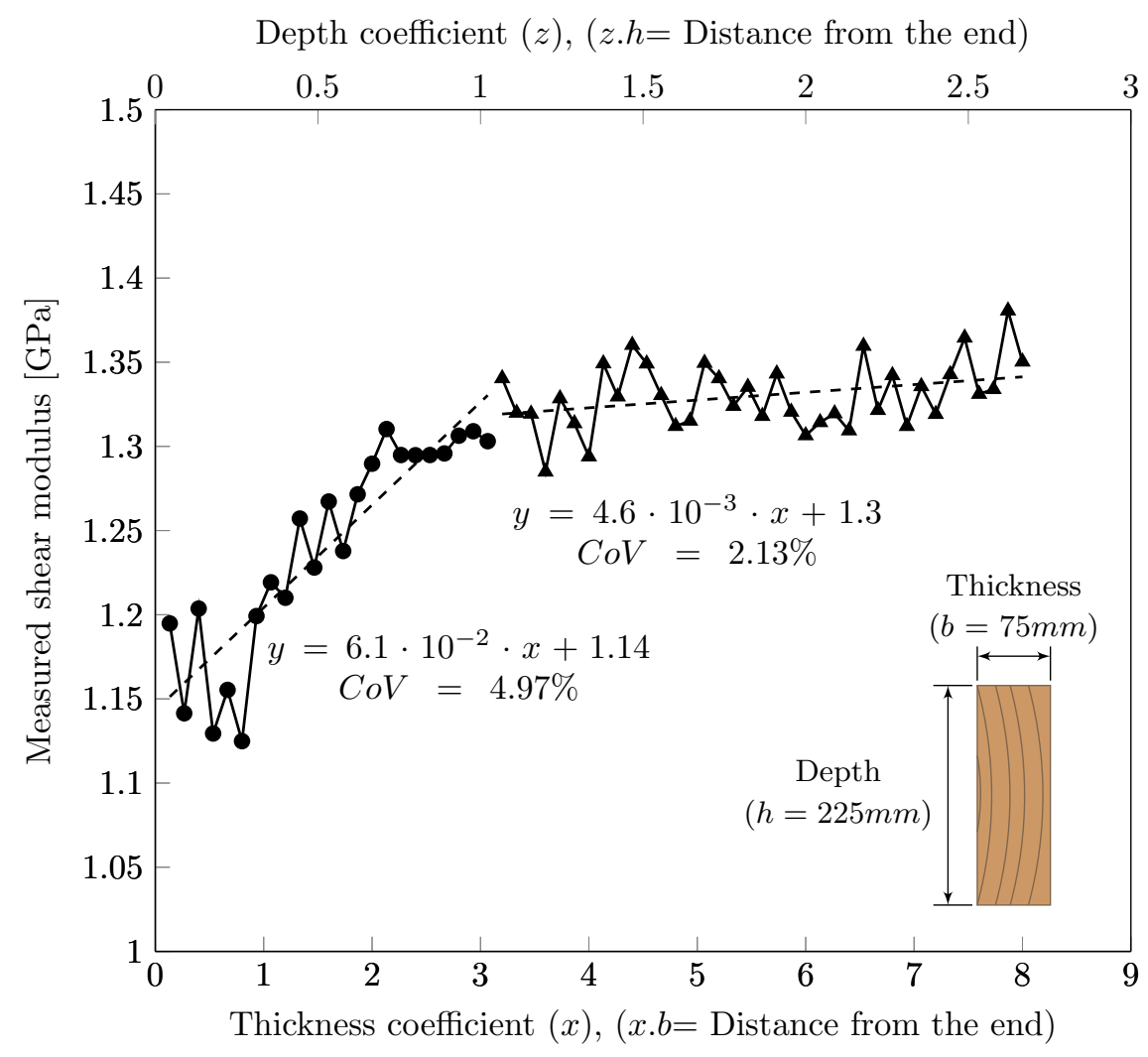

Figure 12: $\mathrm{AR}=3 ; \mathrm{CoV}=5.2 \%$; Spruce 


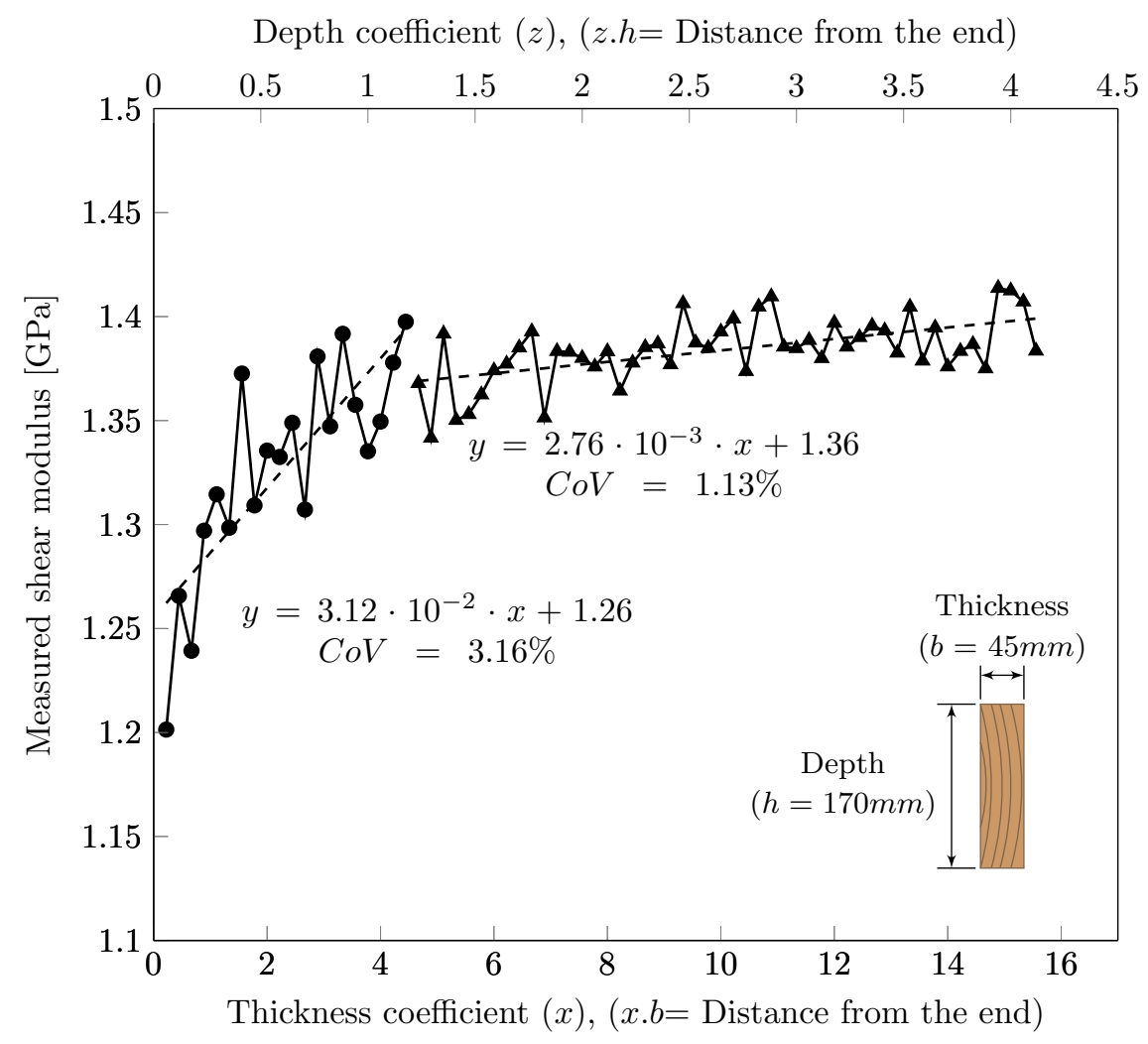

Figure 13: $\mathrm{AR}=3.78$; $\mathrm{CoV}=4.62 \%$; Spruce 


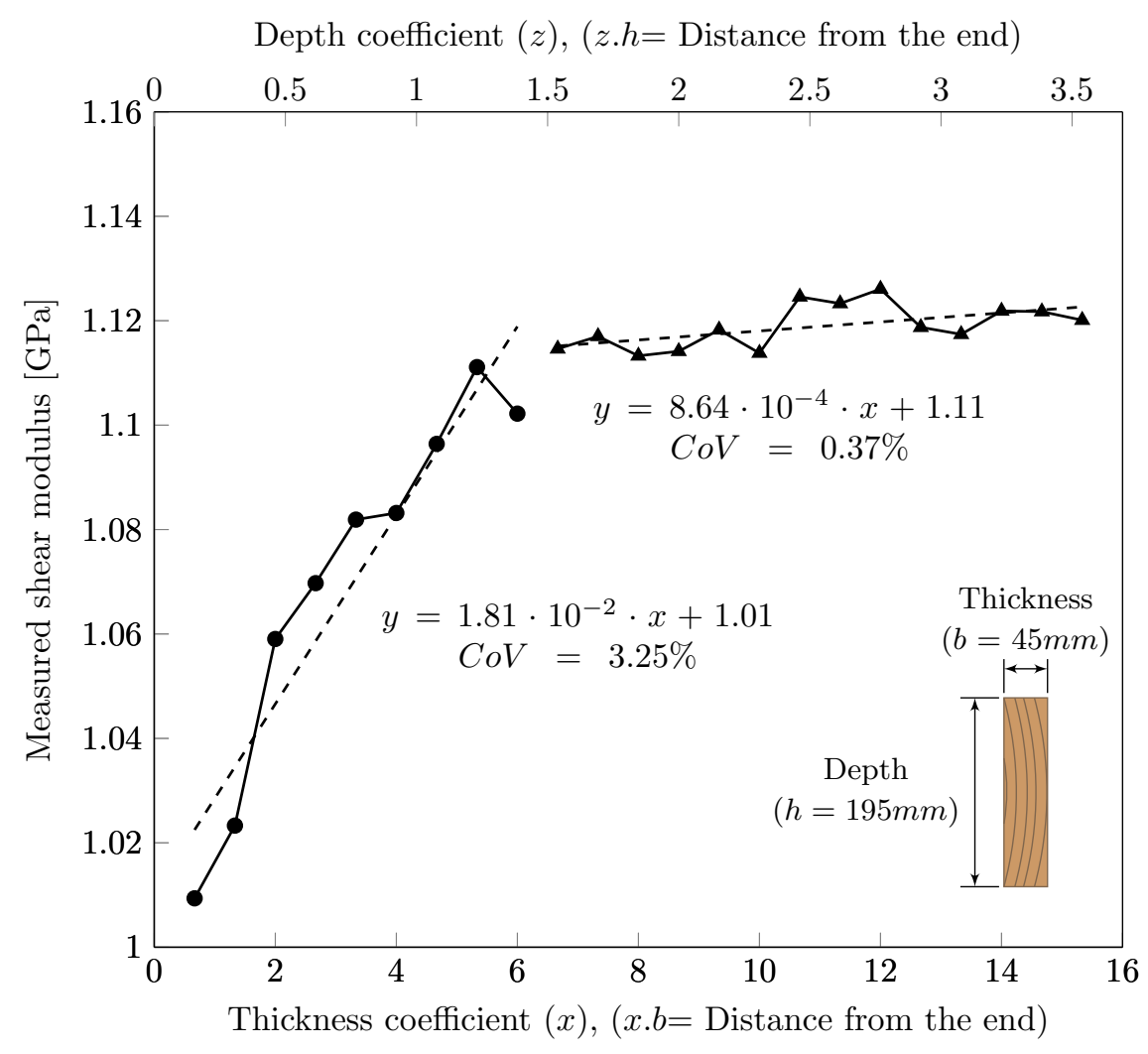

Figure 14: $\mathrm{AR}=4.33 ; \mathrm{CoV}=14.9 \%$; Red Pine 


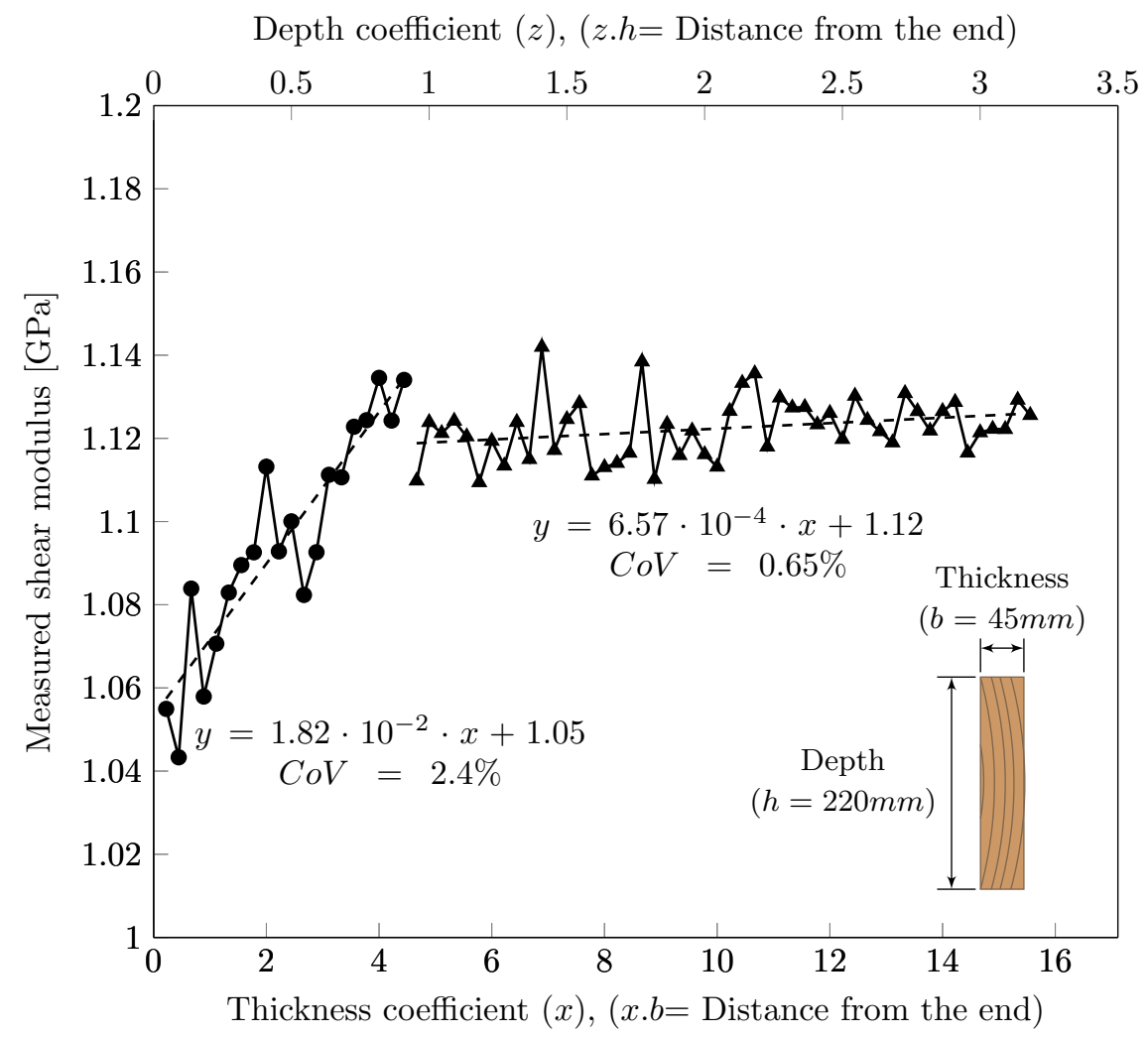

Figure 15: $\mathrm{AR}=4.89$; $\mathrm{CoV}=14.9 \%$; Red Pine 


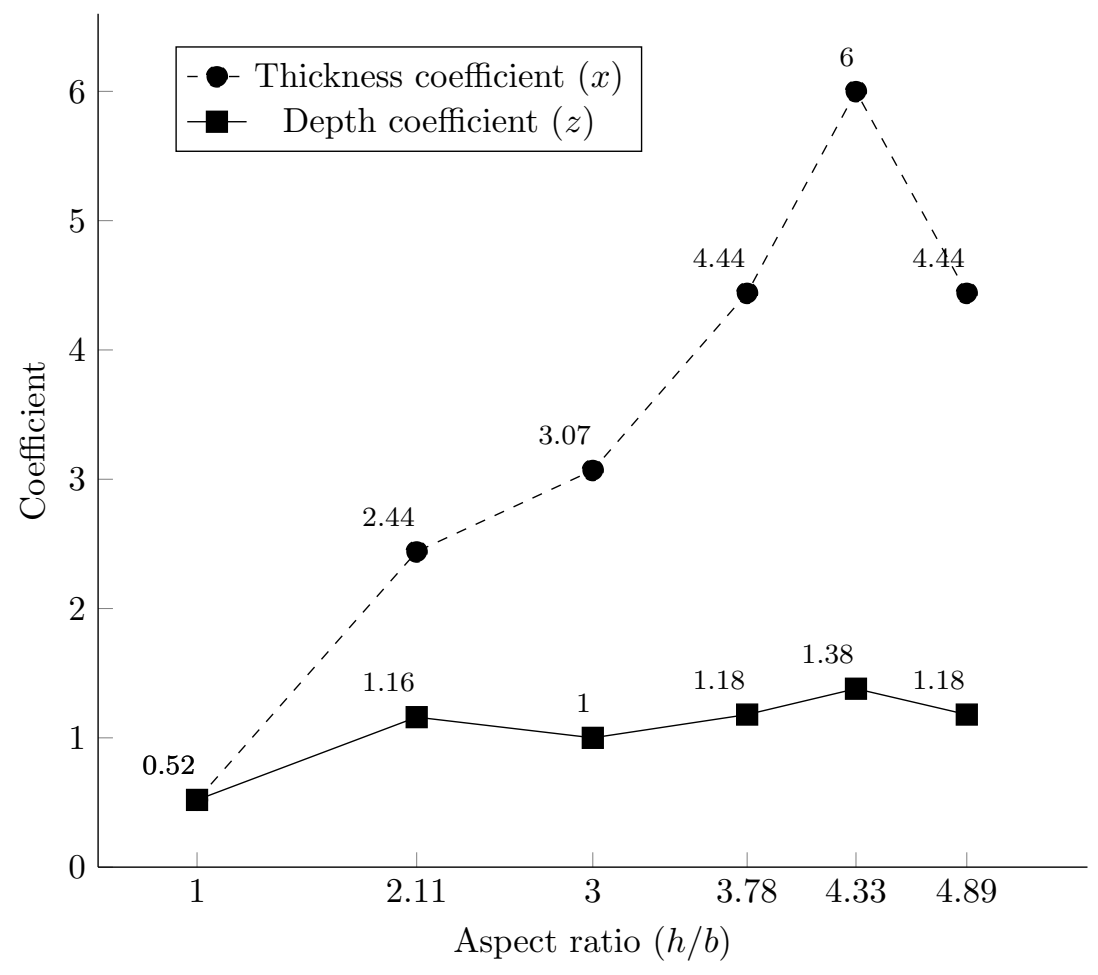

Figure 16: Span of end effect for the tested specimens 
considerably lower in segment two than that in segment one, the length of segment one can be considered as the decay length of the end effect. The length of segment one for each specimen with the different aspect ratios have been collected and converted into the corresponding coefficients of depth and thickness using equations 9 and 10. The results are presented in Figure 16. Although the decay length of the end effect can be estimated using either curve in Figure 16 when an aspect ratio is given, the coefficient of depth is a better parameter when determining the upper bound of the coefficient. Hence, it would be more appropriate to use the depth as the referencing dimension in BS EN 408 (BSI, 2010) rather than the thickness.

$$
\begin{gathered}
\text { Depth coefficient }(z)=\frac{\text { Length of segment one }}{h} \\
\text { Thickness coefficient }(x)=\frac{\text { Length of segment one }}{b}
\end{gathered}
$$

\section{Conclusions and Recommendations}

The following conclusions can be drawn:

1. This research has concluded that the distance between the gauge sections and the supports as specified in the BS EN 408 for the torsion test setup, is not sufficient. This distance is not far enough to avoid the impact of the end effect, which will bring in unnecessary errors in the measurement for the cross sections with high aspect ratio.

2. Contradicted to BS EN 408, our research has indicated that it is more appropriate to use the depth as the referencing dimension to specify the required minimum distance. 
3. Look into the test results of this experimental research, a minimum distance of $1.5 h$ can be observed. This agrees with the previous numerical and analytical studies.

4. The gauges and rotation measuring system illustrated in BS EN 408 are not well designed. The circular gauge may not rotate the same angle as the specimen due to the possible warping in the cross-section. In addition, the LVDTs used in the system will not be able to handle a slightly larger rotations.

5. The proposed non-destructive and non-contact photogrammetry technique has proven to be an efficient yet a precise way of measuring the surface rotation in multiple locations simultaneously.

\section{Acknowledgements}

The authors would like to gratefully acknowledge the financial support provided by Lawrence Ho Research Fund and Peter KK Lee PhD Studentships.

\section{References}

Boresi, A. P., Schmidt, R. J., 2003. Torsion. In: Advanced Mechanics of Materials, 6th Edition. John Wiley and sons, New York, USA, Ch. 6, pp. 200-262.

BSI, 2002. BS EN 13183-1:2002, Moisture Content of a Piece of Sawn Timber. Determination by Oven Dry Method. The British Standards Institution, London, UK. 
BSI, 2010. BS EN 408:2010+A1:2012: Timber structures - Structural Timber and Glued Laminated Timber - Determination of Some Physical and Mechanical Properties. The British Standards Institution, London, UK.

BSI, 2016. BS EN 338:2016: BSI, Structural Timber Strength Classes. The British Standards Institution, London, UK.

Choi, I., Horgan, C. O., 1977. Saint-Venant's Principle and End Effects in Anisotropic Elasticity. Journal of Applied Mechanics 44 (3), 424-430.

URL http://dx.doi.org/10.1115/1.3424095

Davalos, J. F., Qiao, P., Wang, J., Salim, H. a., Schlussel, J., 2002. Shear Moduli of Structural Composites from Torsion Tests. Journal of Composite Materials 36 (10), 1151-1173.

Gupta, R., Heck, L. R., Miller, T. H., 2002a. Experimental Evaluation of the Torsion Test for Determining Shear Strength of Structural Lumber. Journal of Testing and Evaluation 30 (4), 283-290.

URL https://doi.org/10.1520/JTE12318J

Gupta, R., Heck, L. R., Miller, T. H., 2002b. Finite-Element Analysis of the Stress Distribution in a Torsion Test of Full-Size, Structural Lumber. Journal of Testing and Evaluation 30 (4), 291-302.

URL https://doi.org/10.1520/JTE12319J

Gupta, R., Siller, T., 2005. Shear Strength of Structural Composite Lumber 255 using Torsion Tests. Journal of Testing and Evaluation 33 (2), 110-117. URL https://doi.org/10.1520/JTE12287 
Horgan, C., Simmonds, J., 1994. Saint-Venant End Effects in Composite Structures. Composites Engineering 4 (3), 279-286.

URL https://doi.org/10.1016/0961-9526(94)90078-7

260

265

Soltis, L. A., Rammer, D. R., 1994. Shear Strength of Unchecked GluedLaminated Beams. Forest Product Journal 44 (1), 51-57. 
Wohler, C., 2013. 3D Computer Vision: Efficient Methods and Applications, 280 2nd Edition. Springer, London, UK.

URL http://dx.doi .org/10.1007/978-1-4471-4150-1 